\title{
Analysis of risk factors for recurrence after video-assisted pulmonary vein isolation of lone atrial fibrillation-results of 5 years of follow-up
}

\author{
Qiu-Ming Hu, MD, Yan Li, MD, Chun-Lei Xu, MD, Jie Han, MD, Hai-Bo Zhang, MD, Wei Han, MD, and \\ $\mathrm{Xu}$ Meng, MD
}

\begin{abstract}
Objective: The purpose of the present study was to assess the efficacy of the long-term results after videoassisted pulmonary vein isolation and left atrial appendage excision for lone atrial fibrillation (AF) and to determine the most significant risk factors for the long-term results.
\end{abstract}

\begin{abstract}
Methods: From December 2006 to December 2012, 332 consecutive patients with lone AF underwent minimally invasive surgical ablation at our center. Of the 332 patients, 91, who had undergone video-assisted pulmonary vein isolation $>5$ years earlier, were evaluated in the present study (48 with paroxysmal AF, 21 with persistent AF, and 22 with long-standing persistent AF). The median follow-up period was 66 months. The primary endpoint was the success rate of video-assisted pulmonary vein isolation, defined as the absence of any atrial arrhythmia recurrence lasting $>30$ seconds at the clinical visit and on the electrocardiogram or long-term cardiac rhythm recording after discharge.

Results: During the follow-up period, 1 patient (1.1\%) experienced a stroke and $4(4.4 \%)$ died of noncardiac disease. At the 5-year follow-up point, 43 of 78 patients $(55.1 \%)$ were in normal sinus rhythm. Of the 39 patients with paroxysmal AF and 39 with nonparoxysmal AF, 27 (69.2\%) and 16 (44.1\%) were in normal sinus rhythm, respectively. The results of the univariate and multivariate analyses of the preoperative risk factors for AF recurrence showed a left atrial diameter of $\geq 44 \mathrm{~mm}$ (hazard ratio, 5.56; 95\% confidence interval, 1.68-18.387; $P=.005$ ) and an AF duration of $\geq 31.5$ months (hazard ratio, 3.67; 95\% confidence interval, 1.50-8.95; $P=.004)$ were the most significant independent risk factors.
\end{abstract}

Conclusions: Patients with lone AF with a large preoperative left atrial diameter and long AF duration will not be suitable for video-assisted pulmonary vein isolation alone and might need to undergo ablation of the lesions. (J Thorac Cardiovasc Surg 2014;148:2174-80)

Since Haissaguerre and colleagues ${ }^{1}$ reported that ectopic beats originating in the pulmonary veins can result in the spontaneous initiation of AF in 1998, concept development in AF treatment has brought about changes in the technology and methods of AF surgery. In 2005, Wolf and colleagues $^{2}$ performed video-assisted pulmonary vein isolation (PVI) on 27 cases of lone $\mathrm{AF}$ (LAF) and reported that $97 \%$ of the patients were free of AF at early follow-up. Since then, a variety of minimally invasive surgical techniques have been widely applied worldwide. The percentage of success with minimally invasive surgery for $\mathrm{AF}$ has ranged from $42 \%$ to $95.5 \%$ in the published data, with a follow-up period of 6 to 36 months. ${ }^{3-10}$ We retrospectively analyzed the data from patients who had

From the Department of Cardiac Surgery, Beijing Anzhen Hospital of the Capital University of Medical Sciences, Beijing, People's Republic of China.

Disclosures: Authors have nothing to disclose with regard to commercial support.

Received for publication May 1, 2013; revisions received Oct 3, 2013; accepted for publication Oct 29, 2013; available ahead of print March 31, 2014.

Address for reprints: Xu Meng, MD, Department of Cardiac Surgery, Beijing Anzhen Hospital of the Capital University of Medical Sciences, Chaoyang District, Beijing 100029, People's Republic of China (E-mail: mxu@263.net).

$0022-5223 / \$ 36.00$

Copyright (c) 2014 by The American Association for Thoracic Surgery

http://dx.doi.org/10.1016/j.jtcvs.2013.10.054 undergone video-assisted PVI and left atrial appendage (LAA) excision with $>5$ years of follow-up at our center, representing one of the rare experiences with 5 years of follow-up.

\section{METHODS \\ Patient Selection}

From December 2006 to December 2012, 332 patients with LAF underwent minimally invasive surgery at the Atrial Fibrillation Center, Beijing Anzhen Hospital. The 91 patients who had undergone video-assisted PVI $\geq 5$ years earlier were enrolled in the present study. The institutional review board approved the research protocol, and all patients provided informed consent before surgery. We classified all the patients as having either paroxysmal AF (PAF) or non-PAF. The non-PAF group included those with persistent $\mathrm{AF}$ and longstanding, persistent $\mathrm{AF}$. We followed the European Society of Cardiology guidelines to score the AF-related symptoms (European Heart Rhythm Association score). ${ }^{11}$ Preoperative data were collected for each patient enrolled in the present study, including preoperative AF history, body mass index, and $\mathrm{CHADS}_{2}$ score. The patient characteristics are listed in Table 1.

\section{Preoperative Management}

The patients underwent a detailed evaluation before surgery. Baseline 12-lead electrocardiographic (ECG) analysis, chest radiography, transthoracic ultrasound cardiography, transesophageal echocardiography, and computed tomography coronary artery enhancement scanning or coronary angiographic analysis were performed on admission. Also, all the patients 


$$
\begin{aligned}
& \text { Abbreviations and Acronyms } \\
& \text { AF }=\text { atrial fibrillation } \\
& \text { AFL }=\text { atrial flutter } \\
& \text { CI }=\text { confidence interval } \\
& \text { ECG }=\text { electrocardiographic } \\
& \text { EP }=\text { electrophysiologic } \\
& \text { LAA }=\text { left atrial appendage } \\
& \text { LAD }=\text { left atrial diameter } \\
& \text { LAF }=\text { lone AF } \\
& \text { LVDd }=\text { left ventricular end-diastolic dimension } \\
& \text { LVEF }=\text { LV ejection fraction } \\
& \text { PAF }=\text { paroxysmal AF } \\
& \text { PV }=\text { pulmonary vein } \\
& \text { PVI }=\text { PV isolation }
\end{aligned}
$$

were required to complete a health questionnaire about their AF history, previous procedures, antiarrhythmic drug use, preoperative cerebrovascular adverse events, and so on.

\section{Surgical Technique}

We used the surgical technique we have previously reported. ${ }^{12}$ This technique consisted of epicardial radiofrequency isolation of the bilateral pulmonary vein (PV) antrum, excision of the LAA, division of the ligament of Marshall, and electrophysiologic testing. Overall, bilateral PVI was performed using an Isolator Transpolar ENDO ablation clamp (AtriCure, West Chester, Ohio) and the Wolf Lumitip Dissector (AtriCure). LAA removal was performed using the EZ-45 Endostapler (Johnson \& Johnson Medical, Inc, Arlington, Tex). The ligament of Marshall was cut under direct vision. Intraoperative electrophysiologic testing was performed using 2 types of sensing and pacing devices (Detect and Carelink 2090 programmer, Medtronic, Minneapolis, Minn). The procedure was performed using a $10-\mathrm{mm}$, $30^{\circ}$ thoracoscope.

\section{Electrophysiologic Testing}

Intraoperative electrophysiologic (EP) testing was performed in the latest 30 consecutive patients ( 15 with PAF and 15 with persistent AF). EP testing included the bilateral PV antrum and baseline and postisolation sensing and pacing. A baseline positive sensing result (rapid and disorderly atrial potentials) in the PV antrum area could be detected before PVI, and a negative sensing result (no atrial potentials) could be detected in the same area after PVI, which is termed an "entrance block." A positive baseline pacing result was defined as obtaining atrial and ventricular capture. "Capture" has been defined as the contraction of the atrium and ventricle in response to the electrical stimulus sent from the temporary pacemaker. A negative postablation pacing result would indicate that no capture was obtained in the same area after ablation. A combined positive baseline pacing and negative postablation pacing result has been termed "exit block." Achieving both entrance and exit block has been regarded as a transmural lesion blocking conduction in the PV antrum area.

\section{Postoperative Management}

We have recommended that patients take amiodarone in small doses for 3 months postoperatively, with the medicine tapered off in the presence of a stable sinus rhythm. A $\beta$-receptor blocking drug was used for patients intolerant to amiodarone. We have recommended that patients take a vitamin $\mathrm{K}$ antagonist for postoperative anticoagulation in the initial 3-month postoperative period. The international normalized ratio was required to be 2.0 to 3.0. After 3 months, the patients with $\mathrm{AF}$ recurrence or a $\mathrm{CHADS}_{2}$ score $>2$ points with NSR were recommended to take aspirin for anticoagulation. During the follow-up period, direct-current cardioversion was recommended if the ECG analysis showed AF recurrence. Recurrent AF was defined as AF, atrial flutter (AFL), or atrial tachycardia (AT) sustained for $\geq 30 \mathrm{sec}$ onds and occurring out of the postablation blanking period. ${ }^{13,14}$

\section{Follow-up}

Regular follow-up visits were scheduled at 3 and 6 months postoperatively and every year after discharge. At each visit, we offered the patients free physical examinations, 12-lead ECG examinations, and 24- or 48-hour Holter monitoring (Del Mar Reynolds Medical, Inc, Irvine, Calif). During the follow-up period, the primary endpoint was defined as the absence of $\mathrm{AF}$ and any atrial arrhythmia recurrence. In the present study, 63 of the 91 patients $(69.2 \%)$ lived outside of Beijing. It was difficult for these patients to come to our center for each examination. We advised those who could not come to our center to undergo the ECG and echocardiographic examinations in their local city and to mail the results to us. If the patients developed a relapse during the follow-up period, we enquired and recorded the details of the recurrence.

\section{Statistical Analysis}

The patient characteristics and continuous variable data are presented as the mean \pm standard deviation or simple frequencies and percentages. The outcomes of the rhythms were descriptively analyzed at the follow-up intervals. Also, according to the presence of postoperative $\mathrm{AF}$ recurrence during the follow-up period, the patients were classified into 2 groups (nonrecurrence and recurrence). The AF-related risk factors for recurrence between these 2 groups included gender, age, $\mathrm{AF}$ type, $\mathrm{AF}$ duration, left ventricular end-diastolic dimension (LVDd), LV ejection fraction (LVEF), left atrial diameter (LAD), and body mass index. These were included in the univariate analysis using the $t$ test or $\chi^{2}$ test, according to the data type. Risk factors with $P<.1$ on univariate analysis were considered significant to avoid missing more possibly meaningful variables and to relax the variable selection criteria. Receiver operating characteristic curves were used to determine the cutoff value for the continuous variables that were statistically significant on univariate analysis. These continuous variables were classified into dummy variables according to their cutoff value, and their statistically significant differences in each group were tested in Kaplan-Meier curves. The Cox proportional hazards model was used to assessed the risk factors (the dummy variables and the statistically significant categorical variables on univariate analysis). The forward stepwise selection procedure was used. Statistical analyses were performed using the Statistical Package for Social Sciences, version 20.0 (SPSS, Chicago, Ill).

\section{RESULTS \\ Operative Results, Complications, and Mortality}

The mean ablation time was 2.5 hours (range, 2.0-5.5). Of the 91 patients, $75(82.4 \%)$ were in normal sinus rhythm (NSR) after surgery. Before ablation, 1 patient $(1.1 \%)$ underwent permanent pacemaker implantation because of bradyarrhythmia and slow ventricular conduction. Also, 2 patients $(2.2 \%)$ were found to have an unidentified smoke shadow located in the LAA base on the transesophageal echocardiogram preoperatively. To confirm the transesophageal echocardiographic results, the 2 patients underwent computed tomography. The computed tomography results showed the smoke shadow was in a nonenhanced area 
TABLE 1. Baseline patient characteristics before ablation

\begin{tabular}{lc}
\hline \multicolumn{1}{c}{ Characteristic } & Results \\
\hline Gender & \\
Male & 58 \\
Female & 33 \\
Age (y) & $58.0 \pm 9.8$ \\
AF type & \\
$\quad$ PAF & $48(52.7)$ \\
Non-PAF & $43(47.3)$ \\
AF duration (mo) & $70.4 \pm 58.8$ \\
Preoperative AAD use & \\
$\beta$-Blocker & $58(63.7)$ \\
Amiodarone & $50(54.9)$ \\
Digoxin & $20(22.0)$ \\
LVEF* (\%) & $63.0 \pm 9.2$ \\
LVDd* (mm) & $48.1 \pm 5.4$ \\
LAD $\dagger$ (mm) & $49.4 \pm 11.0$ \\
BMI (kg/m ${ }^{2}$ ) & $25.8 \pm 5.6$ \\
Hypertension & $32(35.2)$ \\
Diabetes mellitus & $4(4.4)$ \\
Alcoholic history & $14(15.4)$ \\
Smoke history & $17(18.7)$ \\
Embolic events before ablation & $7(7.7)$ \\
Hyperthyroid & $2(2.2)$ \\
Preoperative permanent pacemaker implantation & $1(1.1)$ \\
Previous failed CA & $2(2.2)$ \\
LAA thrombus & $2(2.2)$ \\
NYHA class I-II & $64(70.3)$ \\
EHRA score III-IV & $44(48.4)$ \\
CHADS ${ }_{2}$ score $<2$ & $61(67.0)$ \\
\hline
\end{tabular}

Data presented as mean \pm standard deviation or $\mathrm{n}(\%) . A F$, Atrial fibrillation; $P A F$, paroxysmal AF; $A D D$, antiarrhythmic drug; $L V E F$, left ventricular ejection fraction; $L V D d$, left ventricular end-diastolic dimension; $L A D$, left atrial diameter; $B M I$, body mass index; $C A$, catheter ablation; $L A A$, left atrial appendage; $N Y H A$, New York Heart Association; EHRA, European Heart Rhythm Association. *Data were collected for 87 patients. $\nmid$ LAD was measured in the parasternal long axis, indicating the left atrial anteroposterior diameter.

with a well-defined margin in the LAA. The distance between the nonenhanced area to the orifice of LAA was $>1$ $\mathrm{cm}$. Because of concerns regarding safety, both patients underwent LAA removal before ablation. Neither patient developed LAA thrombus after LAA excision, and neither developed thromboembolic events after surgery. One patient $(1.1 \%)$ underwent conversion to median sternotomy because of bleeding during right inferior pulmonary vein dissection, early in our experience with this procedure. EP testing was successfully performed in the latest 30 patients, and all 30 patients achieved both entrance and exit block. One patient $(1.1 \%)$ developed acute cardiac dysfunction caused by subendocardial infarction and recovered within 12 days. No patient died in the perioperative period. The mean ventilator support time was $12.9 \pm 6.4$ hours. The mean hospitalization was $15.9 \pm 9.2$ days. The mean follow-up duration was $60.1 \pm 15.8$ months. During the follow-up period, 21 patients $(23.1 \%)$ received direct current cardioversion, 2 of them $(2.2 \%)$ twice. At the latest follow-up point, no patient required a vitamin $\mathrm{K}$ antagonist for anticoagulation. Of the 78 patients followed up for $\geq 5$ years, $38(48.7 \%)$ with $\mathrm{AF}$ recurrence $(46.2 \%, 36$ of 78$)$ or a $\mathrm{CHADS}_{2}$ score $>2$ points with NSR $(2.6 \%, 2$ of 78$)$ received aspirin therapy to prevent cerebrovascular events. One patient $(1.1 \%)$ experienced a postoperative stroke in 3 months after discharge $\left(\mathrm{CHADS}_{2}\right.$ score, 5). Four patients died of noncardiac disease during later follow-up.

\section{Rhythm Results}

During the 5-year period, 9 patients $(9.9 \%)$ had changed their telephone number and home address and thus could not be contacted. In the present study, 63 of 91 patients $(69.2 \%)$ did not live in Beijing. It was difficult for the patients who lived at a distance to come and stay in Beijing for several days to undergo Holter monitoring. We suggested that all patients undergo 12-lead ECG examinations at our center at each follow-up interval. Patients with AF or any other arrhythmia on 12-lead ECG analysis were not asked to undergo Holter analysis again. The Holter monitors were only used for the patients with normal 12-lead ECG findings. In all, 592 interviews were completed during the follow-up period, and 445 Holter monitoring results were recorded. The Holter monitor recording rate was $75.2 \%$ per patient.

At discharge, 66 of the 91 patients $(72.5 \%)$ were in NSR, $21(23.1 \%)$ were in AF, and $4(4.4 \%)$ were AFL/AT. At the 3 -month interval, 72 of the 91 patients $(79.1 \%)$ were in NSR. The proportion of patients in NSR was $85.4 \%$ (41 of 48) in those with PAF and $72.1 \%$ (31 of 43) in those with non-PAF. At the 6-month interval, 73 of 90 patients $(81.1 \%)$ were in NSR: 44 of $47(93.6 \%)$ with PAF and 29 of $43(67.4 \%)$ with non-PAF. At the latest follow-up point, the data were complete for 78 patients, and $43(55.1 \%)$ were in NSR: 27 of $39(69.2 \%)$ with PAF and 16 of 39 $(41.0 \%)$ with non-PAF. Overall, 40 of the 78 patients $(51.3 \%)$ were in sinus rhythm and not taking any antiarrhythmic drugs at 5 years postoperatively. In the present study, no significant difference was found in the proportion of patients in NSR between those who had undergone EP testing and those who had not $(63.3 \%$ vs $55.7 \%, P=.7)$.

The outcomes of the rhythms were descriptively analyzed at each interval during the 5-year follow-up period (Figure 1).

\section{Risk Factors for Later AF Recurrence}

During the follow-up period, the primary endpoint was the success rate of video-assisted PVI, defined as the absence of any atrial arrhythmia recurrence lasting $>30$ seconds at the clinical visit and at ECG analysis or on the long-term cardiac rhythm recording after discharge. We classified all 91 patients into 2 groups according to the occurrence of the primary endpoint events. The independent risk factors for $\mathrm{AF}$ recurrence in these 2 groups were compared (Table 2). The results showed that 6 risk factors 


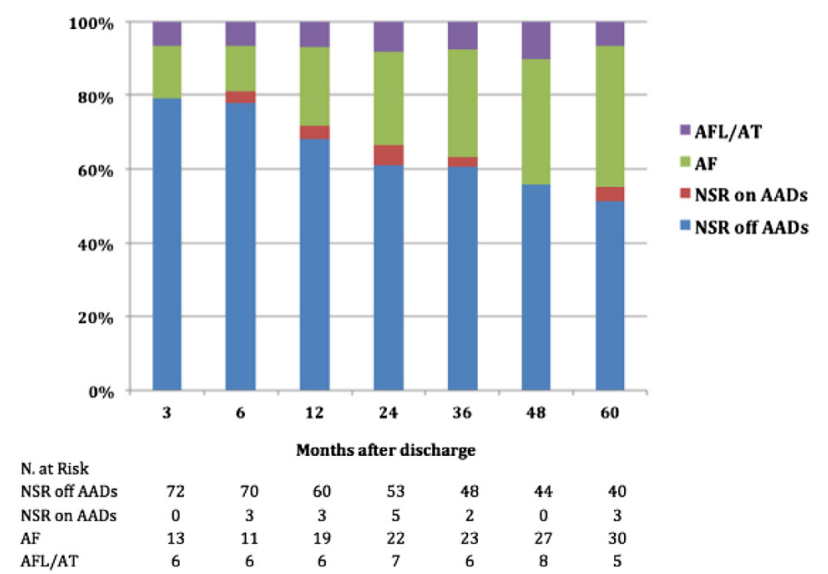

FIGURE 1. General review of heart rhythm at each postoperative period. $A F L / A T$, Atrial flutter/atrial tachycardia; $A F$, atrial fibrillation; $N S R$, normal sinus rhythm; $A A D s$, antiarrhythmic drugs; $N$., number.

had a $P$ value of $<.1$, including gender, non-PAF, AF duration, LVDd, LVEF, and LAD.

From the receiver operating characteristic curve analysis, the preoperative LVEF, LAD, AF duration, and LVDd were predictive of postoperative AF/AFL/AT recurrence (Figure 2). The cutoff point for the LVEF was $64.5 \%$, with a sensitivity of $61.5 \%$ and specificity of $65.7 \%$. The area under the curve was estimated to be 0.69 (95\% confidence interval $[\mathrm{CI}] 0.57-0.80 ; P=.003)$. The optimal cutoff point for the LAD was $44 \mathrm{~mm}$, with a sensitivity of $92.1 \%$ and specificity of $52.8 \%$. The area under the curve was estimated to be 0.77 ( $95 \%$ CI, $0.68-0.86 ; P<.001$ ). The cutoff point for preoperative AF duration was 31.5 months, with a sensitivity of $84.2 \%$ and specificity of $60.4 \%$. The area under the curve was estimated to be 0.73 (95\% CI, 0.63-0.83; $P<.001$ ). The cutoff point for the LVDd was $45.7 \mathrm{~mm}$, with a sensitivity of $83.3 \%$ and specificity of $42.3 \%$ for predicting postoperative AF/AFL/AT recurrence. The area under the curve was estimated to be $0.62(95 \%$ CI, 0.50-0.74; $P=.062)$.

TABLE 2. Comparison of AF-related risk factors stratified by primary endpoint

\begin{tabular}{lccr}
\hline & \multicolumn{2}{c}{ Primary endpoint reached } & \\
\cline { 2 - 3 } \multicolumn{1}{c}{ Factor } & Yes $(\mathbf{n}=\mathbf{5 3})$ & No $(\mathbf{n}=\mathbf{3 8})$ & $\boldsymbol{P}$ value \\
\hline Male gender & $30(56.6)$ & $28(73.7)$ & .095 \\
Age $(\mathrm{y})$ & $58.4 \pm 8.9$ & $57.5 \pm 10.9$ & .684 \\
Non-PAF & $19(35.8)$ & $24(63.2)$ & .010 \\
AF duration $(\mathrm{mo})$ & $44.7 \pm 49.3$ & $74.0 \pm 52.5$ & .008 \\
LVDd $(\mathrm{mm})$ & $47.1 \pm 4.9$ & $49.5 \pm 5.9$ & .043 \\
LVEF $(\%)$ & $65.5 \pm 8.4$ & $59.5 \pm 9.3$ & .002 \\
LAD $(\mathrm{mm})$ & $44.9 \pm 10.1$ & $55.6 \pm 9.2$ & $<.001$ \\
BMI $\left(\mathrm{kg} / \mathrm{m}^{2}\right)$ & $25.0 \pm 3.4$ & $26.8 \pm 7.7$ & .119 \\
EP testing performed & $11(20.8)$ & $19(50.0)$ & .490 \\
\hline Dapronn
\end{tabular}

Data presented as n $(\%)$ or mean \pm standard deviation. $P A F$, Paroxysmal atrial fibrillation; $L V D d$, left ventricular end-diastolic dimension; $L V E F$, left ventricular ejection fraction; $L A D$, left atrial dimension; $B M I$, body mass index; $E P$, electrophysiologic.
We divided all the patients into 2 groups according to the cutoff points for the 4 risk factors and compared the percentage with postoperative NSR using Kaplan-Meier analysis (Figure 3). Statistically significant differences were found in each group. We found that a preoperative LVEF of $\leq 64.5 \%$, LAD of $\geq 44 \mathrm{~mm}$, AF duration of $\geq 31.5$ months, LVDd of $\geq 45.7 \mathrm{~mm}$, and the presence of nonPAF were independent risk factors in our study.

We included these 5 variables in the Cox proportional hazards model. The primary endpoint was AF recurrence, and 38 patients experienced the primary endpoint. The results showed that an LAD of $\geq 44 \mathrm{~mm}$ (hazard ratio, 5.56; 95\% CI, 1.68-18.387; $P=.005$ ), AF duration of $\geq 31.5$ months (hazard ratio, 3.67; 95\% CI, 1.50-8.95; $P=.004$ ) were identified as independent risk factors on multivariate analysis (Table 3).

\section{DISCUSSION}

The initial onset of AF could be related to the mostly ectopic triggers limited to the PVs. ${ }^{15-17}$ However, as the AF duration increases, the AF mechanisms could involve the interaction of many factors. It has been well recognized that the main factors that participate in the formation and maintenance of $\mathrm{AF}$ include triggers limited to the $\mathrm{PV}$, right atrial trigger, autonomic ganglia plexus, and the reentry cycle on the posterior wall of the left atrium. ${ }^{14}$ Thus, videoassisted PVI alone will not be suitable for all patients with AF.

Although our long-term results have shown that the cure rate of minimally invasive PVI alone has been relatively low, it was still greater than the long-term results of single catheter ablation, as recently reported. It was reported by Sorgente and colleagues ${ }^{18}$ that the cure rate for single catheter ablation was only $23 \%$ at 6 years of follow-up. Also, for all the cases, the cure rate for paroxysmal $\mathrm{AF}$ was $36 \%$ and for persistent AF was only $15 \% .{ }^{18}$ Catheter ablation has been limited by its complications, such as PV stenosis, thrombosis, and pericardial tamponade. It also has the disadvantages of a long "learning curve" and poor repeatability. Catheter ablation not only carries a high risk of reoperation, but also increases patients' economic and psychological burden. It has also been demonstrated in a recent double-center randomized clinical study of catheter ablation and minimally invasive surgical ablation of atrial fibrillation that the overall cure rate for catheter ablation was obviously lower than that of minimally invasive surgical ablation during the same period $(36.5 \%$ vs $65.6 \%$, $P=.0022) .{ }^{19}$ Minimally invasive PVI has shown more advantages than catheter ablation in some respects.

In the American Heart Association/European Society of Cardiology AF management guidelines, AF has been classified into PAF, persistent AF, and longstanding, persistent $\mathrm{AF}$ according to the preoperative duration of $\mathrm{AF} .^{11,20} \mathrm{At}$ present, the preoperative AF type has been accepted as an essential index of whether the patient is suitable for 

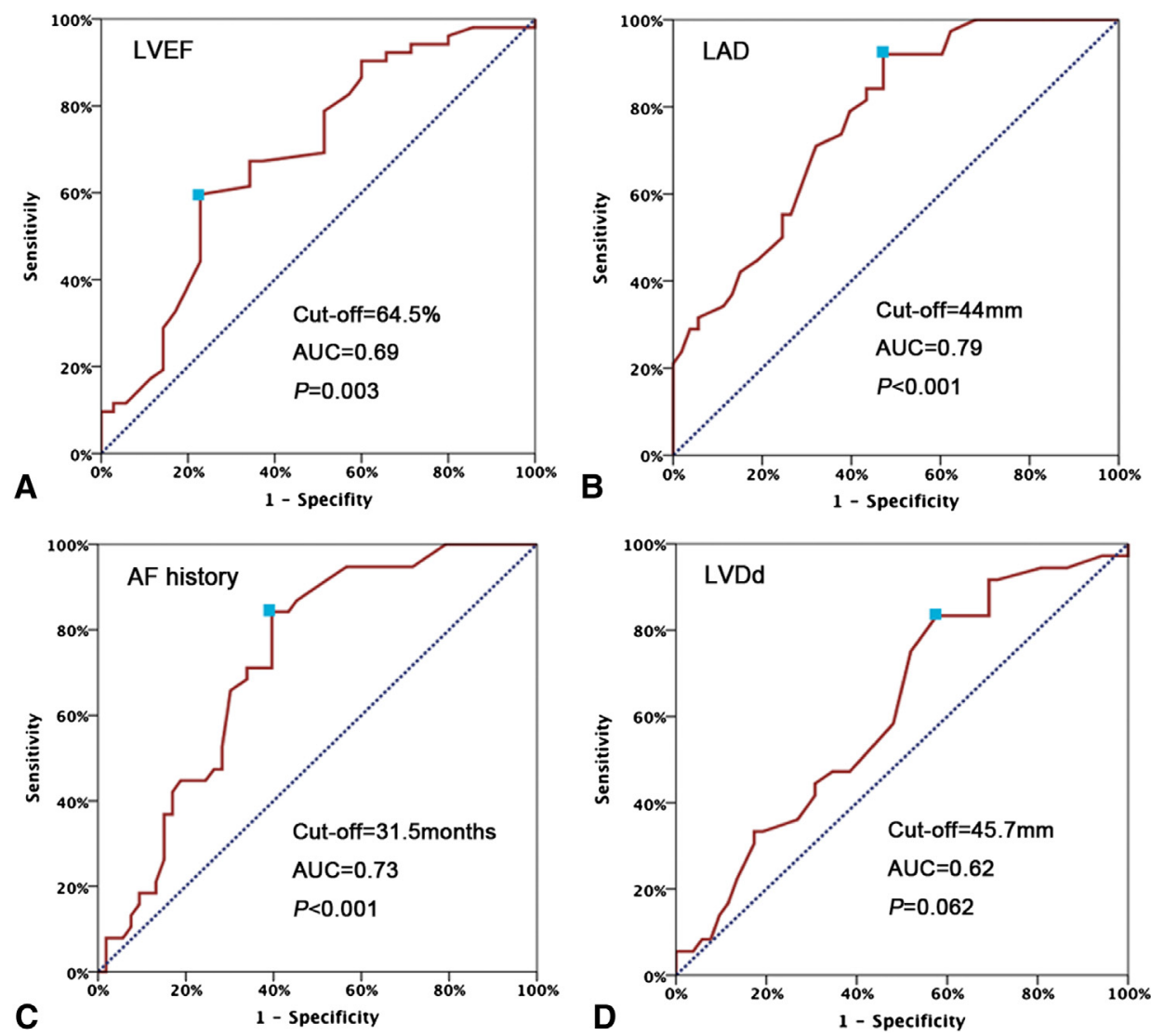

FIGURE 2. Receiver operating characteristics curve showing the cutoff value for preoperative (A) left ventricular ejection fraction ( $L V E F$ ), B, left atrial diameter $(L A D), \mathrm{C}$, atrial fibrillation $(A F)$ history, and $\mathrm{D}$, left ventricular end-diastolic dimension $(L V D d)$ for atrial fibrillation/atrial flutter/atrial tachycardia recurrence after video-assisted pulmonary vein isolation alone. $A U C$, Area under the curve.

video-assisted PVI alone. It has been previously reported that the postoperative NSR rate of PVI alone was greater for PAF, with a significantly lower NSR rate for persistent AF. ${ }^{8,21,22}$ In our study, the average LAD was $49.4 \pm 11.0$ $\mathrm{mm}$. At 5 years of follow-up, the general NSR rate was $55.1 \%$ (43 of 78). The NSR rate for those with PAF and non-PAF was $69.2 \%$ and $41.0 \%$, respectively $(P=.01)$. Our results have also shown that the long-term NSR rate for those with PAF postoperatively was greater than that for the non-PAF. As recently reported, using the Dallas lesion and five-box lesion to add ablation lines on the basis of PVI improved the NSR rate in those with persistent AF in the early follow-up period. ${ }^{23-25}$ This might have resulted from preventing the macroreentry from propagating by adding ablation lines. ${ }^{26}$ The additional ablation lesions were created for patients with LAF and a large preoperative LAD and long AF duration later in our study period. The initial results from our center showed that the cure rate for this novel procedure for non-PAF was $75.0 \%$ at 1 year of follow-up. Our results have also demonstrated that PVI alone might not be sufficient for persistent AF.

However, the question remains whether all patients with PAF are suitable for video-assisted PVI alone and whether all patients with persistent AF are not suitable for videoassisted PVI alone. In some studies, the risk factors that could affect the postablation recurrence of AF also included the preoperative LAD, ablation device, and energy selection. ${ }^{27-30}$ The results of our study showed that a preoperative AF duration of $\geq 31.5$ months was another independent risk factor for $\mathrm{AF}$ recurrence. A total of 53 patients had an AF duration of $\geq 31.5$ months $(47.2 \%$ with PAF and $52.8 \%$ with non-PAF) and 38 had an AF duration of $<31.5$ months $(60.5 \%$ with PAF and $39.5 \%$ with nonPAF). Our results have shown that the NSR of patients with an AF duration of $\geq 31.5$ months was significantly lower than that of patients with an AF duration of $<31.5$ months $(39.6 \%$ vs $84.2 \% ; P<.001)$. When we included the preoperative AF type, AF duration of $\geq 31.5$ months, non-PAF, and preoperative LVDd, LVEF, and LAD in the Cox proportional hazards model, a preoperative AF duration of $\geq 31.5$ months and LAD were statistically significant, with the LAD the most essential risk factor (hazard ratio, 5.56; $95 \%$ CI, $1.68-18.387 ; P=.005)$. This might indicate that the preoperative LAD is more sensitive than the preoperative AF duration for predicting the recurrence of AF postoperatively. In addition, the average LAD of the patients 

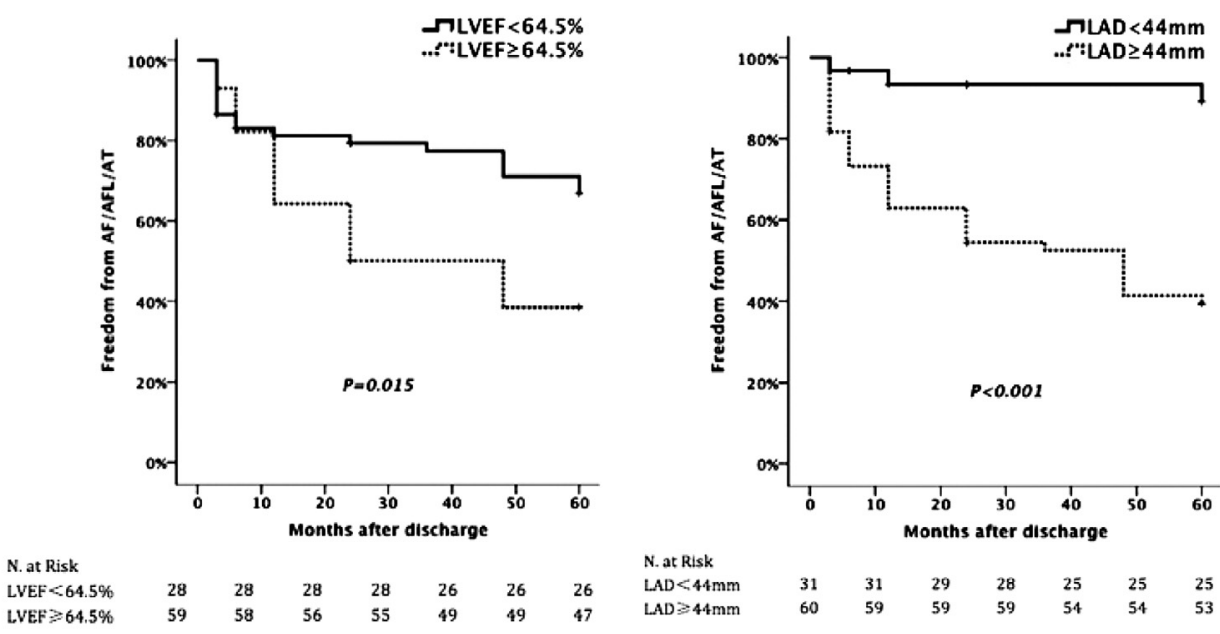

A
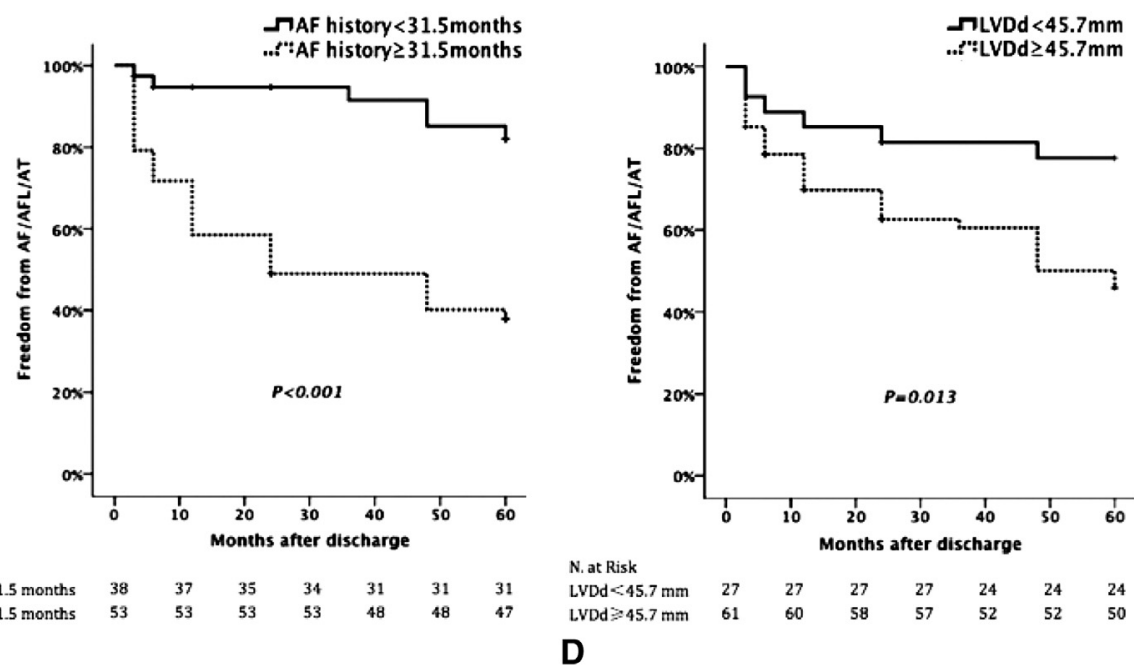

FIGURE 3. Kaplan-Meier postoperative atrial fibrillation $(A F)$-free survival between the patients with preoperative (A) left ventricular ejection fraction $(L V E F)$ of $<64.5 \%$ versus $\geq 64.5 \%(P=.015)$; B, left atrial diameter $(L A D)$ of $<44 \mathrm{~mm}$ versus $\geq 44 \mathrm{~mm}(P<.001)$; C, atrial fibrillation duration of $<31.5$ months versus $\geq 31.5$ months $(P<.001)$; and $\mathrm{D}$, left ventricular end-diastolic dimension $(L V D d)$ of $<45.7 \mathrm{~mm}$ versus $\geq 45.7 \mathrm{~mm}(P=.013)$.

with $\mathrm{AF}$ and an $\mathrm{AF}$ duration of $\geq 31.5$ months versus an $\mathrm{AF}$ duration of $<31.5$ months was $52.8 \pm 10.9 \mathrm{~mm}$ and $44.6 \pm$ $9.4 \mathrm{~mm}(P<.001)$, respectively. This finding could also have demonstrated that as the duration of $\mathrm{AF}$ increases, the left atrium expands and affects the postoperative recurrence of AF. These findings could also have resulted because as the hemodynamics change (greater atrial pressure and wall stress), the structural remodeling would be worse (fibrosis, dilatation, and hypertrophy) in the left atrium, ${ }^{31}$ which could

TABLE 3. Hazard ratios for recurrence in Cox proportional hazards model

\begin{tabular}{lllc}
\hline \multicolumn{1}{c}{ Factor } & HR & \multicolumn{1}{c}{$\mathbf{9 5} \%$ CI } & $\boldsymbol{P}$ value \\
\hline $\mathrm{LAD} \geq 44 \mathrm{~mm}$ & 5.56 & $1.68-18.39$ & .005 \\
$\mathrm{AF}$ duration $\geq 31.5 \mathrm{mo}$ & 3.67 & $1.50-8.95$ & .004 \\
\hline
\end{tabular}

$H R$, Hazard ratio; $C I$, confidence interval; $L A D$, left atrial diameter; $A F$, atrial fibrillation. lead to the facilitation of AF recurrence. As recently reported, the occurrence of postoperative AFL/AT was mainly related to reentry around the ablation lines in the PVs, posterior wall of the left atrium, and isthmus. ${ }^{32}$

Our study had several limitations. The small sample size in our study might have limited the generalizability and caused the wide range of CIs for the LAD in the multivariate model. In the early stage of beginning this technique in our clinic center, we lacked the device and technology for EP testing; thus, two thirds of patients did not undergo EP testing. Also, most of our patients did not live in Beijing, and it was difficult for them to come and stay in Beijing for several days to undergo Holter monitoring. We suggested that all patients undergo 12-lead ECG examinations at our center at each follow-up interval. The patients who were in AF or had any other arrhythmia by the 12-lead ECG analysis were not recommended to undergo Holter 
analysis again. The Holter monitors were used only for those patients with normal 12-lead ECG findings. Thus, our Holter monitoring results could not reflect the true situation. As a retrospective study, our study also was limited by the lack of a comparison with catheter ablation or other surgical energy sources and technologies.

\section{CONCLUSIONS}

Our research has shown that the postoperative NSR rate after video-assisted PVI alone would be low for patients with $\mathrm{AF}$ and a larger LAD and long AF duration. The LAD was the most important factor determining longterm postoperative recurrence. In addition, for the patients with a longer AF duration, the rate of recurrence was greater, and video-assisted PVI alone should not be recommended for those patients. In contrast, video-assisted PVI alone could be considered for patients with a shorter AF duration and a normal LAD. At present, we have performed 332 minimally invasive surgeries in our center. Additional ablation lesions were created for the patients with LAF and a large preoperative LAD and long AF duration in our later study. The later results will be verified as our study continues.

\section{References}

1. Haissaguerre M, Jais P, Shah DC, Takahashi A, Hocini M, Quiniou G, et al. Spontaneous initiation of atrial fibrillation by ectopic beats originating in the pulmonary veins. N Engl J Med. 1998;339:659-66.

2. Wolf RK, Schneeberger EW, Osterday R, Miller D, Merrill W, Flege JB Jr, et al. Video-assisted bilateral pulmonary vein isolation and left atrial appendage exclusion for atrial fibrillation. J Thorac Cardiovasc Surg. 2005;130:797-802.

3. Pruitt JC, Lazzara RR, Dworkin GH, Badhwar V, Kuma C, Ebra G. Totally endoscopic ablation of lone atrial fibrillation: initial clinical experience. Ann Thorac Surg. 2006;81:1325-30; discussion 1330, 1321.

4. Edgerton JR, Edgerton ZJ, Weaver T, Reed K, Prince S, Herbert MA, et al. Minimally invasive pulmonary vein isolation and partial autonomic denervation for surgical treatment of atrial fibrillation. Ann Thorac Surg. 2008;86:35-8; discussion 39.

5. Wudel JH, Chaudhuri P, Hiller JJ. Video-assisted epicardial ablation and left atrial appendage exclusion for atrial fibrillation: extended follow-up. Ann Thorac Surg. 2008;85:34-8.

6. Beyer E, Lee R, Lam BK. Point: minimally invasive bipolar radiofrequency ablation of lone atrial fibrillation: early multicenter results. J Thorac Cardiovasc Surg. 2009;137:521-6.

7. Lee AM, Clark K, Bailey MS, Aziz A, Schuessler RB, Damiano RJ Jr. A minimally invasive Cox-maze procedure: operative technique and results. Innovations. 2010;5:281-6.

8. Yilmaz A, Geuzebroek GS, Van Putte BP, Boersma LV, Sonker U, De Bakker JM, et al. Completely thoracoscopic pulmonary vein isolation with ganglionic plexus ablation and left atrial appendage amputation for treatment of atrial fibrillation. Eur J Cardiothorac Surg. 2010;38:356-60.

9. La Meir M, Gelsomino S, Luca F, Pison L, Chambille P, Parise O, et al. Timerelated prevalence of postoperative atrial fibrillation after stand-alone minimally invasive radiofrequency ablation. J Card Surg. 2011;26:453-9.

10. Edgerton ZJ, Edgerton JR. Rationale for minimally invasive pulmonary vein isolation and partial autonomic denervation for surgical treatment of atrial fibrillation. Innovations. 2008;3:121-4.

11. European Heart Rhythm Association, European Association for Cardio-Thoracic Surgery, Camm AJ, Kirchhof P, Lip GY, Schotten U, Savelieva I, Ernst S, et al. Guidelines for the management of atrial fibrillation: the task force for the management of atrial fibrillation of the European Society of Cardiology (ESC). Eur Heart J. 2010;31:2369-429.
12. Cui YQ, Li Y, Gao F, Xu CL, Han J, Zeng W, et al. Video-assisted minimally invasive surgery for lone atrial fibrillation: a clinical report of 81 cases. J Thorac Cardiovasc Surg. 2010;139:326-32.

13. European Heart Rhythm Association, European Cardiac Arrhythmia Society, American College of Cardiology, American Heart Association, Society of Thoracic Surgeons, Calkins H, Brugada J, Packer DL, Cappato R, Chen SA, Crijns HJ, et al. HRS/EHRA/ECAS expert consensus statement on catheter and surgical ablation of atrial fibrillation: recommendations for personnel, policy, procedures and follow-up. A report of the Heart Rhythm Society (HRS) task force on catheter and surgical ablation of atrial fibrillation. Heart Rhythm. 2007;4:816-61.

14. Calkins H, Kuck KH, Cappato R, Brugada J, Camm AJ, Chen SA, et al. 2012 HRS/EHRA/ECAS expert consensus statement on catheter and surgical ablation of atrial fibrillation: recommendations for patient selection, procedural techniques, patient management and follow-up, definitions, endpoints, and research trial design. J Intervent Cardiac Electrophysiol. 2012;33:171-257.

15. Jais P, Haissaguerre M, Shah DC, Chouairi S, Gencel L, Hocini M, et al. A focal source of atrial fibrillation treated by discrete radiofrequency ablation. Circulation. 1997;95:572-6.

16. Shiroshita-Takeshita A, Brundel BJ, Nattel S. Atrial fibrillation: basic mechanisms, remodeling and triggers. J Intervent Cardiac Electrophysiol. 2005;13:181-93.

17. Drewitz I, Steven D, Lutomsky B, Servatius H, Willems S, Rostock T. Persistent, isolated pulmonary vein re-entry: inducibility, entrainment, and overdrive termination of a sustained tachycardia within an isolated pulmonary vein. Europace. 2008;10:261-4.

18. Sorgente A, Tung P, Wylie J, Josephson ME. Six year follow-up after catheter ablation of atrial fibrillation: a palliation more than a true cure. Am J Cardiol. 2012;109:1179-86.

19. Boersma LV, Castella M, van Boven W, Berruezo A, Yilmaz A, Nadal M, et al. Atrial fibrillation catheter ablation versus surgical ablation treatment (FAST): a 2-center randomized clinical trial. Circulation. 2012;125:23-30.

20. Wann LS, Curtis AB, January CT, Ellenbogen KA, Lowe JE, Estes NA III, et al; ACCF/AHA Task Force Members. 2011 ACCF/AHA/HRS focused update on the management of patients with atrial fibrillation (updating the 2006 guideline): a report of the American College of Cardiology Foundation/American Heart Association task force on practice guidelines. Circulation. 2011;123:104-23.

21. Han FT, Kasirajan V, Kowalski M, Kiser R, Wolfe L, Kalahasty G, et al. Results of a minimally invasive surgical pulmonary vein isolation and ganglionic plexi ablation for atrial fibrillation: single-center experience with 12-month followup. Circulation. 2009;2:370-7.

22. Edgerton JR, McClelland JH, Duke D, Gerdisch MW, Steinberg BM, Bronleewe SH, et al. Minimally invasive surgical ablation of atrial fibrillation: six-month results. J Thorac Cardiovasc Surg. 2009;138:109-13; discussion 114.

23. Edgerton JR, Jackman WM, Mahoney C, Mack MJ. Totally thoracoscopic surgical ablation of persistent $\mathrm{AF}$ and long-standing persistent atrial fibrillation using the "Dallas" lesion set. Heart Rhythm. 2009;6:S64-70.

24. Edgerton JR, Jackman WM, Mack MJ. A new epicardial lesion set for minimal access left atrial maze: the Dallas lesion set. Ann Thorac Surg. 2009;88:1655-7.

25. Sirak J, Jones D, Schwartzman D. The five-box thoracoscopic maze procedure. Ann Thorac Surg. 2010;90:986-9.

26. Lockwood D, Nakagawa H, Peyton MD, Edgerton JR, Scherlag BJ, Sivaram CA, et al. Linear left atrial lesions in minimally invasive surgical ablation of persistent atrial fibrillation: techniques for assessing conduction block across surgical lesions. Heart Rhythm. 2009;6:S50-63.

27. Lo LW, Tai CT, Lin YJ, Chang SL, Udyavar AR, Hu YF, et al. Predicting factors for atrial fibrillation acute termination during catheter ablation procedures: implications for catheter ablation strategy and long-term outcome. Heart Rhythm. 2009;6:311-8.

28. La Meir M, Gelsomino S, Luca F, Lorusso R, Gensini GF, Pison L, et al. Minimally invasive thoracoscopic hybrid treatment of lone atrial fibrillation: early results of monopolar versus bipolar radiofrequency source. Interact Cardiovasc Thorac Surg. 2012; 14:445-50.

29. McCarthy PM, Kruse J, Shalli S, Ilkhanoff L, Goldberger JJ, Kadish AH, et al. Where does atrial fibrillation surgery fail? Implications for increasing effectiveness of ablation. J Thorac Cardiovasc Surg. 2010;139:860-7.

30. Cheema A, Vasamreddy CR, Dalal D, Marine JE, Dong J, Henrikson CA, et al. Long-term single procedure efficacy of catheter ablation of atrial fibrillation. $J$ Intervent Cardiac Electrophysiol. 2006;15:145-55.

31. Burstein B, Nattel S. Atrial fibrosis: mechanisms and clinical relevance in atrial fibrillation. J Am Coll Cardiol. 2008;51:802-9.

32. Lee G, Sanders P, Kalman JM. Catheter ablation of atrial arrhythmias: state of the art. Lancet. 2012;380:1509-19. 